\title{
Expression of the Baculovirus p35 Protein in Tobacco Affects Cell Death Progression and Compromises $N$ Gene-Mediated Disease Resistance Response to Tobacco mosaic virus
}

\author{
Olga del Pozo and Eric Lam \\ Biotech Center, Foran Hall, 59 Dudley Road, Rutgers State University of New Jersey, New Brunswick, NJ 08903, U.S.A. \\ Submitted 10 October 2002. Accepted 31 December 2002.
}

\begin{abstract}
The $\mathbf{p 3 5}$ protein from baculovirus is a broad-range caspase inhibitor and suppresses programmed cell death in animals. We report here the effects of transgenic expression in tobacco of the p35 protein during the hypersensitive response (HR). Expression of p35 causes partial inhibition of nonhost HR triggered by bacteria and gene-for-gene $H R$ triggered by virus. Infection of p35-expressing tobacco plants with Tobacco mosaic virus (TMV) disrupts $N$-mediated disease resistance, causing systemic spreading of the virus within a resistant background. Mutant variants altered in aspartate residues within the loop region of $\mathbf{p 3 5}$ are inefficient substrates for caspases in vitro, and they do not suppress caspase proteolytic activity in animal systems. Tobacco plants expressing these mutant variants of the p35 protein do not show inhibition of HR cell death or enhanced virus systemic movement. Thus, HR inhibition and TMV systemic spreading phenotype in p35-expressing plants correlate with the ability of the p35 protein to suppress caspase activity in animal systems. In addition, a Cterminal truncated variant of $\mathrm{p35}$ is unable to suppress cell death in animals as well as HR cell death in transgenic tobacco. Our results provide evidence for the participation of caspase-like proteases during the HR. In addition, they suggest that timely activation of cell death is necessary for effective TMV containment within the primary infection site.
\end{abstract}

Additional keywords: caspase-like proteins.

Programmed cell death (PCD) in plants takes place during development and after pathogen challenge (Greenberg 1996). The host success in the plant-pathogen battle relies on the ability of plant cells to detect the pathogen presence rapidly. In many cases, this recognition also triggers a rapid cell death in host tissues at and surrounding the entry point of the pathogen. This cell death is accompanied by the synthesis of antimicrobial and protective compounds, which contribute to pathogen restriction at the primary site of infection. This complex process is called the hypersensitive response (HR) and takes place when paired genes of avirulence ( $A v r$ ) from the pathogen and resistance $(R)$ from the host are present, being both at the apex of the immediate cascade

Corresponding author: Eric Lam: E-mail: Lam@aesop.rutgers.edu.

Current address for Olga del Pozo: Boyce Thompson Institute for Plant Research, Tower Road, Ithaca, NY 14853, U.S.A. of events that follow (Mittler and Lam 1996; Morel and Dangl 1997). When the plant is able to mount a successful HR, long term systemic defense responses take place and protect the plant against future pathogen infection, constituting the systemic acquired resistance (SAR) phenomenon (Yang et al. 1997). Lack of the $A v r$ or the $R$ gene product results in the failure of early detection of the pathogen, and the battle is won on the pathogen side, which then systemically colonize the infected plant. Although development of a macroscopic HR is often associated with the onset of pathogen resistance, examples in which these phenotypes can be uncoupled have also been reported (e.g., the Arabidopsis dndl mutant, in which macroscopic HR is dramatically suppressed while resistance to certain bacterial pathogens is maintained [Yu et al. 1998]). Also, different genetic elements in the interspecies hybrid Nicotiana edwardsonii, originating from the two parental lines, have been shown to be independently responsible for the activation of $\mathrm{HR}$ and resistance to Cauliflower mosaic virus (CaMV) (Cole et al. 2001). Thus, the relevance of cell death during the HR to disease resistance may depend on the particular pathogen and its interaction with the host.

PCD is a common process in multicellular organisms during development and in response to pathogens and stress signals (Raff 1998). Research from numerous laboratories during the last decade led to the identification of molecules that constitute the death machinery in different animal model systems. Among those, caspases are conserved aspartate-specific cysteine proteases that play a central role in signaling and executing PCD in phylogenetically diverse multicellular organisms (Cryns and Yuan 1998). Database searches (Aravind et al. 1999; Uren et al. 2000) led to the identification of putative proteins that may contain caspase-related domains in bacteria, fungi, and plants. No structural homologue of caspase has been reported in plants, although some of the components of the animal apoptotic machinery show homology to participants of the resistance response in plants to pathogens (Van der Biezen and Jones 1998). Recently, proteolytic activity with similarities to caspase has been detected in plants during HR cell death (del Pozo and Lam 1998; Tian et al. 2000).

Caspase inhibitors have been widely used for demonstration of caspase participation and for caspase activity characterization in cell death process of different systems. There are different types of caspase inhibitors, synthetic inhibitors, endogenous cellular inhibitors, and viral physiological inhibitors, which include the IAP and p35 proteins from baculovirus and the CrmA protein from cowpox virus (Kidd 1998). Among them, p35 is a well-characterized specific inhibitor of caspases. The $\mathrm{p} 35$ protein itself is a substrate for caspases, and cleavage 
at Asp87 is necessary for stable p35-caspase complex formation and inhibition of caspase activity. When expressed ectopically, p35 protein is able to suppress cell death in different animal models that are phylogenetically distant (Clem et al. 1996). The p35 protein crystal structure revealed a reactive soluble loop that contains the caspase cleavage site located at the apex of the loop, fully accessible for the target enzymes (Fisher et al. 1999). Kinetic and substrate characterization of p35 (Zhou et al. 1998) showed that it is a very specific inhibitor for all known caspases and has no detectable inhibitory effect on other types of proteases.

We previously demonstrated that coinfiltration of synthetic caspase inhibitors with HR-inducing bacteria in tobacco results in a dramatic inhibition of HR cell death (del Pozo and Lam 1998). Moreover, caspase-like protease activity could be detected in tobacco leaf extracts from tissues that were developing HR cell death after virus infection. p35 protein has been used frequently as a tool to demonstrate caspase involvement in animal apoptosis in vivo. Following this approach and in order to examine the physiological consequence of HR cell death suppression, we generated transgenic tobacco plants expressing baculovirus p35 protein and studied bacteria- and virus-induced HR cell death. Here, we report that p35-expressing plants showed partial inhibition of HR cell death compared with wild-type (WT) tobacco plants. Importantly, transgenic tobacco plants expressing mutant versions of the $\mathrm{p} 35$ protein did not show this phenotype. A striking characteristic of p35-expressing transgenic plants is that TMV is able to escape from the primary infected leaves and infect systemically the inoculated plant, in spite of the presence of the $N$ resistance gene. Thus, in this particular plant-virus interaction, timely induction of HR cell death is necessary for disease resistance and pathogen restriction to the primary infection site. Plants expressing p35 phenocopy the recently described dominant negative mutants of the $N$ gene (Dinesh-Kumar et al. 2000) and represent a novel system to study the dynamics of host-virus interaction in a resistant background. Our results demonstrate
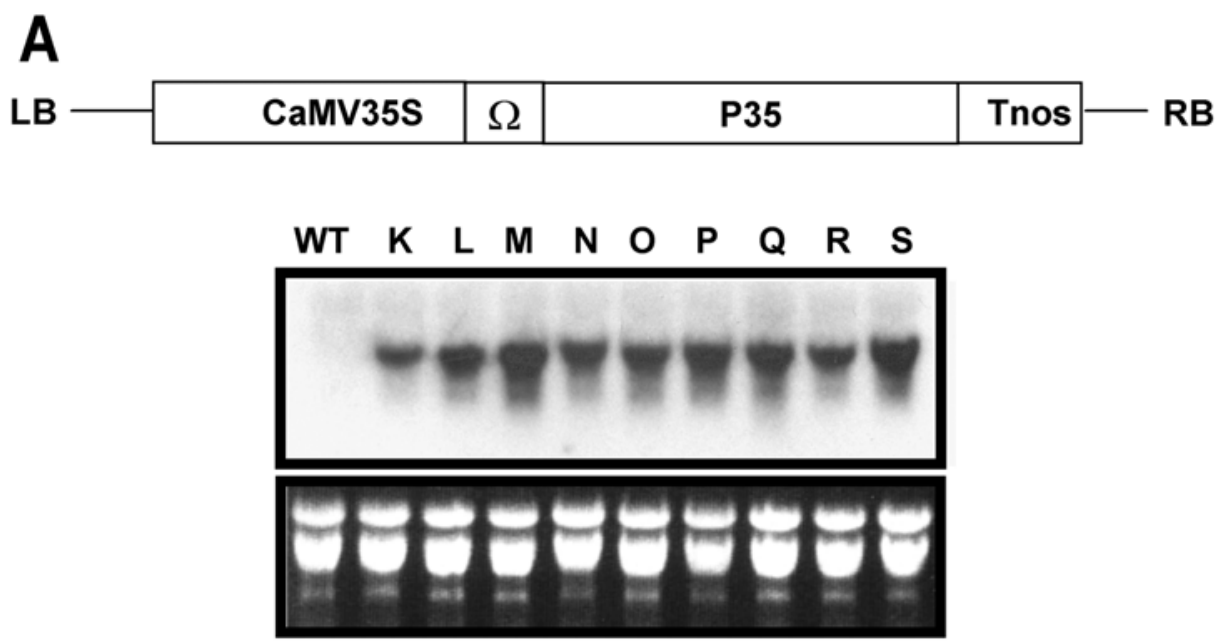

B

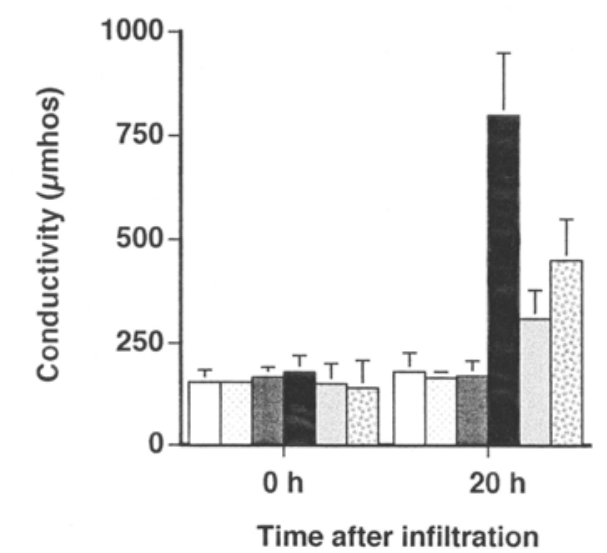

WT + NPS 4000

p35-O + NPS 4000

$\mathrm{p} 35-\mathrm{Q}+\mathrm{NPS} 4000$

WT + NPS 3121

p350 + NPS 3121

p35Q + NPS 3121

Fig. 1. Transgenic p35-expressing tobacco plants show delayed hypersensitive response (HR) cell death upon incompatible bacteria infiltration. A, Scheme of the T-DNA binary vector in which the p35 coding region was inserted between the omega translation-enhancing region and the nos $3^{\prime}$ polyadenylation signal (nopaline synthase transcription termination sequence). This vector was used to transform tobacco plants, and the level of p35 expression in independent transgenic tobacco lines was compared by Northern blot analysis, using the p35 coding sequence as a probe. Each line was designated by a letter shown above the lanes and the nontransformed plant shown as wild type (WT). B, Infiltration of p35-expressing plants with HR-inducing bacteria (NPS 3121). Measurement of ion leakage with leaf discs from WT tobacco plants and two independent p35-expressing lines, p35-O and p35-Q. The data represent the mean and the standard error of means of three individual measurements corresponding to three different plants per tobacco line. 
that the $\mathrm{p} 35$ protein from baculovirus affects the rate of HR cell death in tobacco after pathogen challenge. The fact that a nonhost HR and a gene-for-gene HR are affected by the expression of the p35 protein suggests that both HR pathways share a similar downstream component in the cell death signaling and execution pathway that might be a caspase-like protein.

\section{RESULTS}

Expression of baculovirus p35 protein in tobacco delays HR cell death.

We generated transgenic tobacco plants expressing the p35 protein from baculovirus. The p35 coding sequence was inserted

A
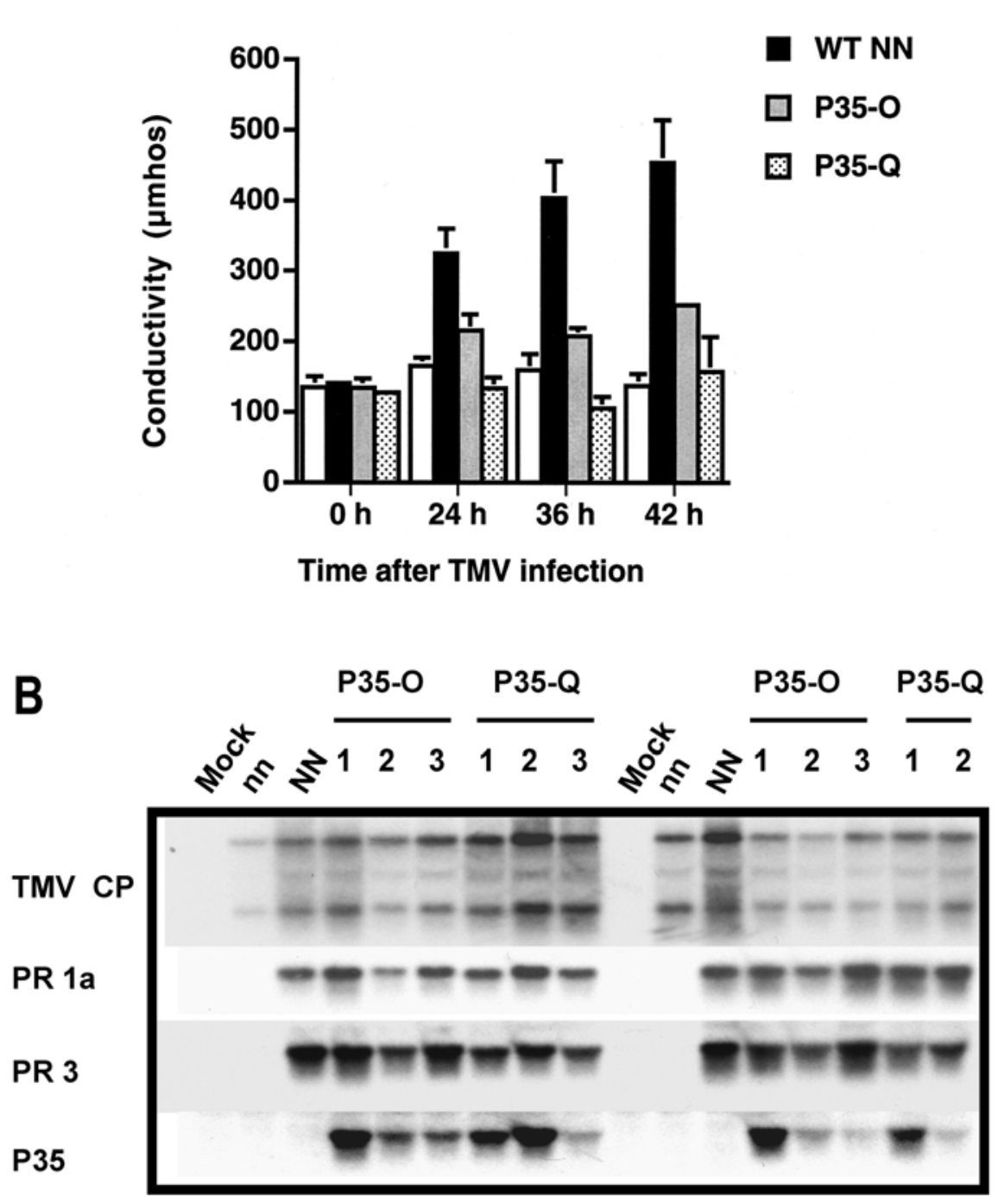

\section{days after infection}

\section{loading} control
Time after TMV infection mock 
into a binary vector immediately downstream from the constitutive $35 \mathrm{~S}$ promoter from CaMV and the omega sequence of TMV for enhanced translation. Northern blots with RNA isolated from independent lines of transgenic tobacco plants confirmed high levels of expression of the p35 transcript (Fig. 1A). Plants expressing p35 presented a normal phenotype and were able to produce flowers and seeds. In order to analyze p35 effects on HR cell death, we infiltrated p35-expressing plants with Pseudomonas syringae pv. phaseolicola NPS 3121, a bean pathogen that induces nonhost HR in this species when infiltrated at high titer (Lindgren et al. 1986), and quantitated the relative progress of cell death by measuring cellular electrolyte leakage (conductivity) at different times after infiltration. At late stages of plant cell death, cellular contents leak into the intercellular space, since there is no engulfment of cellular debris by neighboring cells as in animal apoptosis, due to the presence of the plant cell wall. This ion leakage can be used to compare the relative amount of cell death in plant tissues. As a negative control, we performed similar infiltrations with an isogenic $P$. syringae pv. phaseolicola strain that is $\mathrm{Hrp}^{-}$(NPS 4000) and is unable to trigger the HR. Cell death in p35-expressing plants infiltrated with HR-inducing bacteria (NPS 3121) was partially inhibited compared with WT plants (Fig. 1B). Visually, the HR lesions of WT plants appeared more "desiccated" than those observed with p35 transgenic plants, although the whole infiltrated area collapsed in both cases. The fact that p35 suppresses the HR only partially compared with the total suppression displayed by synthetic caspase inhibitors is discussed below.

We also examined the response of p35 transgenic plants to TMV infection. Resistant tobacco plants $(N N)$ infected with TMV normally develop HR lesions 24 to $30 \mathrm{~h}$ after viral infection. Tobacco lines expressing p35 showed less HR cell death, as measured by electrolyte leakage than WT tobacco plants, even at $42 \mathrm{~h}$ after infection when lesions were fully developed (Fig. 2A). Nevertheless, there was not a dramatic visual difference between HR-lesions in transgenic and control plants. The lesions were similar in shape, size, number, and time of appearance, although lesions frequently looked less desiccated than in WT plants. To test the possibility that p35 expression was affecting the replication of TMV, we analyzed the relative replication rate of the virus indirectly by Northern blot analysis (Fig. 2B). The rate of TMV accumulation was similar between WT and transgenic plants, since similar increases of TMV coat protein (CP) RNA could be detected in WT and p35-expressing lines at $24 \mathrm{~h}$ and at earlier timepoints after infection (data not shown). At later times (3 days after TMV challenge), consistently less TMV accumulation was observed in primary inoculated leaf tissues of the p35-expressing plants (up to $50 \%$ decrease). However, this is unlikely to be the cause for the delay in HR cell death observed at the early timepoints (Fig. 2A) and may reflect the systemic movement of TMV out of the primary infected tissues in the p35-expressing plants (discussed below). The onset of the local host defense mechanisms in response to TMV challenge was also examined by analyzing the induction of pathogenesis-related (PR) gene expression at 0 to $72 \mathrm{~h}$. We found that PR-1a and PR-3 transcripts could be detected at similar times and levels after virus inoculation of WT or p35 transgenic tobacco plants (Fig. 2B, only the data for 2 and 3 days postinfection (dpi) are shown).

\section{Tobacco plants expressing mutant versions of the $\mathbf{p 3}$ protein are not affected in their HR.}

To determine if the effects observed in p35-expressing plants were due to inhibition of one or more putative plant caspaselike proteins, we generated different mutant versions of the $\mathrm{p} 35$ protein and expressed them in transgenic tobacco lines. The D87A p35 mutant (p35D87A), in which the aspartate residue at the P1 position within the caspase target site has been mutated to alanine, suppressed the antiapoptotic activity of p35 in $C$. elegans, although its cleavage by caspases was not completely abolished (Xue and Horvitz 1995) (Fig. 3). We generated another mutant version of the p35 protein $(\mathrm{p} 35 \mathrm{~m})$, in which Asp81, Asp84, and Asp87 were all mutated into alanine residues by site-directed mutagenesis (Fig. 3A). This mutant version of $\mathrm{p} 35$, according to our hypothesis, should not be cleaved by caspases (discussed below). A 12-amino acid C-terminal deletion of p35 (p35SpeI) also abolishes its antiapoptotic activity in insect cells (Hershberger et al. 1992). We synthesized $\left[{ }^{35}\right.$ S]methionine-labeled p35, p35m, p35D87A, and p35SpeI proteins in vitro and subjected them to cleavage by purified caspase-1. As shown in Figure 3B, p35m cleavage could not be detected $(<3 \%)$, but p35D87A was still cleaved to a significant extent (12\%), although at a lower efficiency than wild-type p35 $(63 \%)$ and p35SpeI (68\%). This result shows that the triple aspartate mutant we have constructed is a more complete null control than the D87A mutant of p35. On the other hand, the SpeI carboxy-terminus deletion mutant of p35 was cleaved as effectively as WT p35 (Fig. 3B).

We generated transgenic tobacco plants that expressed each of the three mutant forms of the p35 protein. Tobacco plants expressing the $\mathrm{C}$-terminal truncation or the triple aspartate mutant p35 derivatives showed similar electrolyte leakage to that observed in WT tobacco plants after TMV challenge (Fig. 3C). Interestingly, the p35D87A mutant is able to provide some protection against PCD during HR in transgenic tobacco plants, as manifested by less electrolyte leakage (Fig. 3D). This is likely due to the presence of aspartate residues in the vicinity of D87 (D81 and D84) that may be used as alternative cleavage sites (Fig. 3B), and thus, the D87A mutant may retain some inhibitory activities for putative plant caspase-like proteases. In the experiment shown in Figure 3D, p35D87A plants expressed slightly higher levels of p35 transcript compared with p35 plants, as determined by Northern blot analysis (data not shown). In addition, the degree of inhibition on electrolyte leakage correlates with the level of p35 expression at the RNA level in p35D87A plants. Thus, the highest expressing line, p35D87A-K, also showed the most dramatic delay in the ion leakage assay. Similar results were obtained upon induction of nonhost HR cell death by $P$. syringae pv. phaseolicola NPS 3121 instead of TMV challenge (data not shown). These results are consistent with our interpretation that the effects of p35 expression on TMV-induced cell death are related to its ability to inhibit caspases. In an effort to rule out the possibility that the lack of cell death inhibition was due to instability of the p35 mutant versions, we generated transgenic tobacco plants that expressed p35-6His, p35m-6His, p35D87A-6His, and p35SpeI$6 \mathrm{His}$ tagged proteins to improve protein detection. Hexahistidine tagging of p35 at the C-terminus does not interfere with p35 binding to caspases and, therefore, does not affect its antiapoptotic function (Fisher et al. 1999). The p35 protein expression level in transgenic tobacco plants with similar transcript levels of the various forms of p35 was analyzed by Western blot using monoclonal antibodies directed against the hexahistidine tag. We detected a very faint band of similar low intensity with protein extracts from a transgenic plant expressing p35 and different p35 mutant forms that were not present in WT extracts (data not shown). This observation suggests that protein stability in transgenic p35 mutants is not altered dramatically compared with $\mathrm{p} 35$-expressing plants.

\section{TMV can systemically infect p35-expressing tobacco plants upon HR cell death suppression.}

In the $N$ gene-TMV system, the HR developed in the host may be instrumental in disease resistance to restrict TMV to 
the initial sites of infection, preventing virus systemic spread (Whitman et al. 1994). Interestingly, many of the p35-expressing plants generated in the $N$ background showed systemic symptoms of TMV infection (vein clearing and stunting of leaves above the inoculated ones) and systemic HR (SHR)-like lesions became visible on various aerial parts of the plant, including the stem, petioles, and younger leaves (Fig. 4A). The frequency of this phenotype was between 50 to $75 \%$ among high p35 expressors (data from three independent experiments, using three individual siblings derived from two independent p35-expressing lines). Symptoms appeared as soon as 4 dpi in young leaves near the shoot apex, after virus challenge on lower, mature leaves (Fig. 4A). In very virulent cases, after two weeks to one month postinfection, the apical meristem and young leaves died due to massive cell death leading to the demise of the complete plant. In less virulent cases, intermediate phenotypes could be observed, and plants remained alive for several months, developing healthy-looking lower lateral shoots that were apparently free of virus. But in all the cases observed, the virus was able to infect and colonize these shoots later on. In cases of slow virus colonization, the shoots were able to produce seeds despite the virus spreading to floral tissues. In these cases, flowers developed HR-like lesions in petals, sepals (Fig. 4B), stamen filaments, and carpels (data not shown). In other cases, the flowers were not able to produce seeds, since the complete flower bud collapsed from massive



Time after TMV infection
B

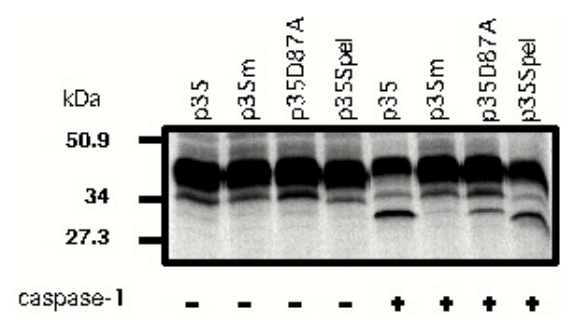

D
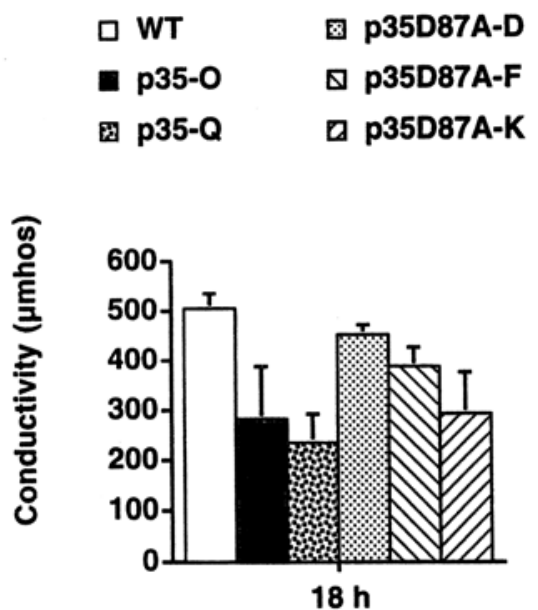

Time after TMV infection

Fig. 3. Analysis of p35 mutations to correlate caspase inhibitory activity with in planta effects. A, p35 structure, caspase cleavage site, and description of the different mutant p35 variants generated. B, [35S]-methionine-labeled p35, p35m, p35D87A, and p35SpeI proteins were incubated with (+) or without (-) $10 \mathrm{ng}$ of purified caspase- 1 for $30 \mathrm{~min}$ at $30^{\circ} \mathrm{C}$ and then were analyzed by $12 \%$ protein gel electrophoresis and autoradiography. $\mathbf{C}$, Infection of transgenic p35, p35m, and p35SpeI plants with Tobacco mosaic virus (TMV). Wild-type (WT) tobacco plants and transgenic plants p35 (p35-O), p35m (p35m-A and p35m-H), and p35SpeI (p35SpeI-I) were infected with TMV, and samples were collected at 0 and $36 \mathrm{~h}$ after TMV infection for ion leakage measurement. Similar results were obtained in two other independent trials, and comparable levels of p35 transcripts were detected by Northern blot analysis in transgenic plants expressing either $\mathrm{p} 35$ or the $\mathrm{p} 35$ mutants (data not shown). $t$-Test results $[\mathrm{P}(\mathrm{T}<=\mathrm{t})]$ comparing WT $N N$ plants versus p35-O transgenic tobacco plants equal 0.046. Comparing p35-O plants versus each transgenic plants expressing different p35 mutants individually gave the following values: $\mathrm{p} 35 \mathrm{~m}-\mathrm{A}=0.035, \mathrm{p} 35 \mathrm{~m}-\mathrm{H}=0.043$, and $\mathrm{pSpeI}-\mathrm{I}=0.083$. D, Effect of the D87A mutation on $\mathrm{p} 35$ inhibition of cell death in tobacco. WT tobacco plants, two independent lines of p35-expressing plants ( $\mathrm{p} 35-\mathrm{O}$ and $\mathrm{p} 35-\mathrm{Q}$ ), and three independent lines of p35D87A-expressing plants (p35D87A-D, p35D87A-F, and p35D87A-K) were infected with Tobacco mosaic virus, and samples were collected at 0 and $18 \mathrm{~h}$ postinoculation for ion leakage measurement. Similar results were obtained in two other independent trials. p35 transcript levels were slightly higher in p35D87A-D and p35D87A-F and about twofold higher in p35D87A-K compared with the level of p35 transcripts in p35 expressing lines p35-O and p35-Q. 
HR cell death (Fig. 4B). Occasionally, curling and wrinkling of the tissue (Fig. 4B, center) preceded lesions. These plants showed stunting and slower growth compared with uninfected p35 plants.

The SHR-lesions in TMV-infected, p35-expressing plants correlates with TMV presence, as determined by Western blot using TMV CP antibodies (Fig. 5A). Virus extracted from these tissues was able to trigger a normal HR response in WT $N N$ tobacco plants, and TMV was localized to the primary infected foci. This result rules out the possibility that selection of a resistance-breaking mutation of the virus is the cause of our observations. In p35-expressing plants, low levels of TMV were also detected in systemic chlorotic areas that were not developing lesions and that resembled tissues from susceptible $n n$ tobacco plants (Fig. 5B). This observation raised the possibility that a certain threshold of TMV accumulation may be necessary for triggering the SHR. Under this critical level, the plant cell is not able to respond to the pathogen and fails to activate cell death. $P R$ gene expression was also monitored in systemically infected regions. Regions showing SHR cell death expressed high levels of PR-1a and PR-3 transcripts (Fig. 5B) and proteins (data not shown). Chlorotic tissues also expressed $P R$ genes, although at lower levels. Thus, the level of PR expression correlated with virus concentration. Transgenic tobacco plants expressing mutants of $\mathrm{p} 35$ protein (p35SpeI or $\mathrm{p} 35 \mathrm{~m}$ ) did not show this TMV-spreading phenotype, although very high expressors of p35D87A-expressing plants (with RNA levels 5 to 10 times higher than p35 plants) did to some extent (data not shown). These results provide correlative evidence that shows the breakdown of $N$-mediated resistance leading to systemic virus infection caused by $\mathrm{p} 35$ expression is likely due to the inhibitory effects on caspase-like proteases in plants.

\section{Tissue distribution of HR-like lesions}

\section{in TMV systemically-infected p35 transgenic plants.}

Large stem epidermal lesions were occasionally observed in p35 plants that showed SHR (Fig. 4C). This raised the question whether the virus was moving from cell to cell within the epidermal layer and eventually developing HR in some areas or whether it was able to enter the vascular tissue and was spreading through the phloem. Systematic microscopic studies of p35-expressing plants were performed to examine these possibilities. In some cases, sectioning showed HR-like lesions within the vascular tissue (Fig. 4D), demonstrating that the virus is able to enter and replicate within the vascular tissue. Surprisingly, sections of stems from shoots in which the epidermis looked healthy and apparently free of virus showed browning in internal tissues that resembled HR-like lesions (Fig. 4D and L). Within these HR-like lesions, the cell types as well as the size and stage of development of the lesion varied along the stem. Higher magnification of brown cells showed deposition of material within the remains of the cells. These cells were reabsorbed eventually and pushed by neighboring cells, resulting in punctated regions within the pith (Fig. 4L, M, and N). In some cases, massive collapse of tissues led to formation of large holes within the pith (Fig. 4E). Browning areas resembling HR-like lesions correlated with TMV presence, as demonstrated by tissue printing and Western blotting using TMV $\mathrm{CP}$ antisera (Fig. 4H, I, J, and K). Large amounts of virus can be localized in and around the lesions, but virus could also be detected (albeit at lower levels) in tissues that did not show any apparent browning phenotype at that time (red arrows in Fig. $4 \mathrm{H}, \mathrm{J}$, and K). No TMV CP protein was detected in distal stem sections of WT $N N$-infected plants (data not shown). HR-like lesion development was accompanied by deposition of fluorescent materials at the periphery (Fig. $4 \mathrm{~F}$ and G). This autofluo- rescence could be detected around and not within the browning areas formed of fully collapsed cells (Fig. 4F and G).

\section{DISCUSSION}

In this paper, we confirmed our previous results with synthetic peptide inhibitors for caspases (del Pozo and Lam 1998) by an in vivo approach, using transgenic plants that express the baculovirus p35 caspase inhibitor. This approach ruled out possible nonspecific effects due to relatively high concentrations of peptide inhibitors for caspases that were required (Schotte et al. 1999). We show that p35-expressing transgenic tobacco plants displayed partial inhibition of cell death associated with nonhost and gene-for-gene HR upon bacteria or virus challenge. The altered cell death phenotype shown by p35-expressing plants is likely dependent on the ability of this protein to inhibit caspases. Indeed, no other target or catalytic function has been reported for $\mathrm{p} 35$, aside from its interaction with and inhibition of various caspases described so far (Zhou et al. 1998). We have previously shown that coinfiltration of NPS 3121 with caspase-specific inhibitors in tobacco results in dramatic inhibition of macroscopic cell death (del Pozo and Lam 1998). The partial suppression of HR cell death in the case of p35-expressing plants may be due to low levels of p35 protein present in the transgenic plants, thus limiting the stoichiometric complex formation with caspases (Bump et al. 1995). P35 protein accumulation in p35-expressing plants assayed by Western blots, using antibodies that recognize p35 and monoclonal antibodies against the hexa-histidine tag (data not shown), was extremely low. This result was not expected, given the amount of p35 mRNA detected in these transgenic plants. Possible explanations for the low levels of p35 protein accumulation include poor translation due to codon bias and stability of p35 protein in plant cells.

\section{Effects of p35 in plants: Mutational studies correlate suppression of $\mathrm{HR}$ cell death to efficacy of caspase inhibition.}

Structural studies revealed that caspases are inhibited in the active site through a covalent thioester linkage to p35 at Asp87. The cleavage of p35 protein by caspases between Asp 87 and Gly88 repositions the N-terminus of p35 into the active site of the protease, eliminating solvent accessibility to the catalytic dyad and maintaining the covalent thioester linkage (Xu et al. 2001). This data validates previous biochemical studies that show cleavage of the p35 protein appears to be necessary for stable complex formation and inhibition of caspases (Bump et al. 1995; Xue and Horvitz 1995). Expression of p35D87A was shown to suppress the antiapoptotic activity of $\mathrm{p} 35$ in C. elegans, although its cleavage by caspases was not completely abolished (Xue and Horvitz 1995) (Fig. 3). A possible interpretation is that the neighboring aspartate residues within this region ${ }_{78} \mathrm{LER}_{\mathrm{D} Y S} \underline{\mathrm{DQMDG}} \underline{\mathrm{D}}_{88}$; D81 and D84) may be used as alternate thioester donor/cleavage sites, albeit at lower efficiencies (Fig. 3A). In order to generate a p35 variant that would be completely resistant to caspase cleavage, we converted Asp81, Asp84, and Asp87 of p35 into alanines (p35m). Indeed, in vitro characterization showed that $\mathrm{p} 35 \mathrm{~m}$ was no longer a substrate for caspase-1 (Fig. 3B). Mutational analysis of the p35 protein showed that charge to noncharge amino acid substitutions within the reactive loop (other than P1-P4) did not alter the capacity of p35 to inhibit caspases (Bertin et al. 1996). Thus, replacement of aspartate residues by alanines in the soluble loop of $\mathrm{p} 35$ protein should cause minimal perturbation in its overall structure.

Transgenic tobacco plants were constructed, in which different mutant p35 coding sequences (p35m, p35D87A, and p35SpeI) were expressed. Plants expressing $\mathrm{p} 35 \mathrm{~m}$ protein developed a HR 

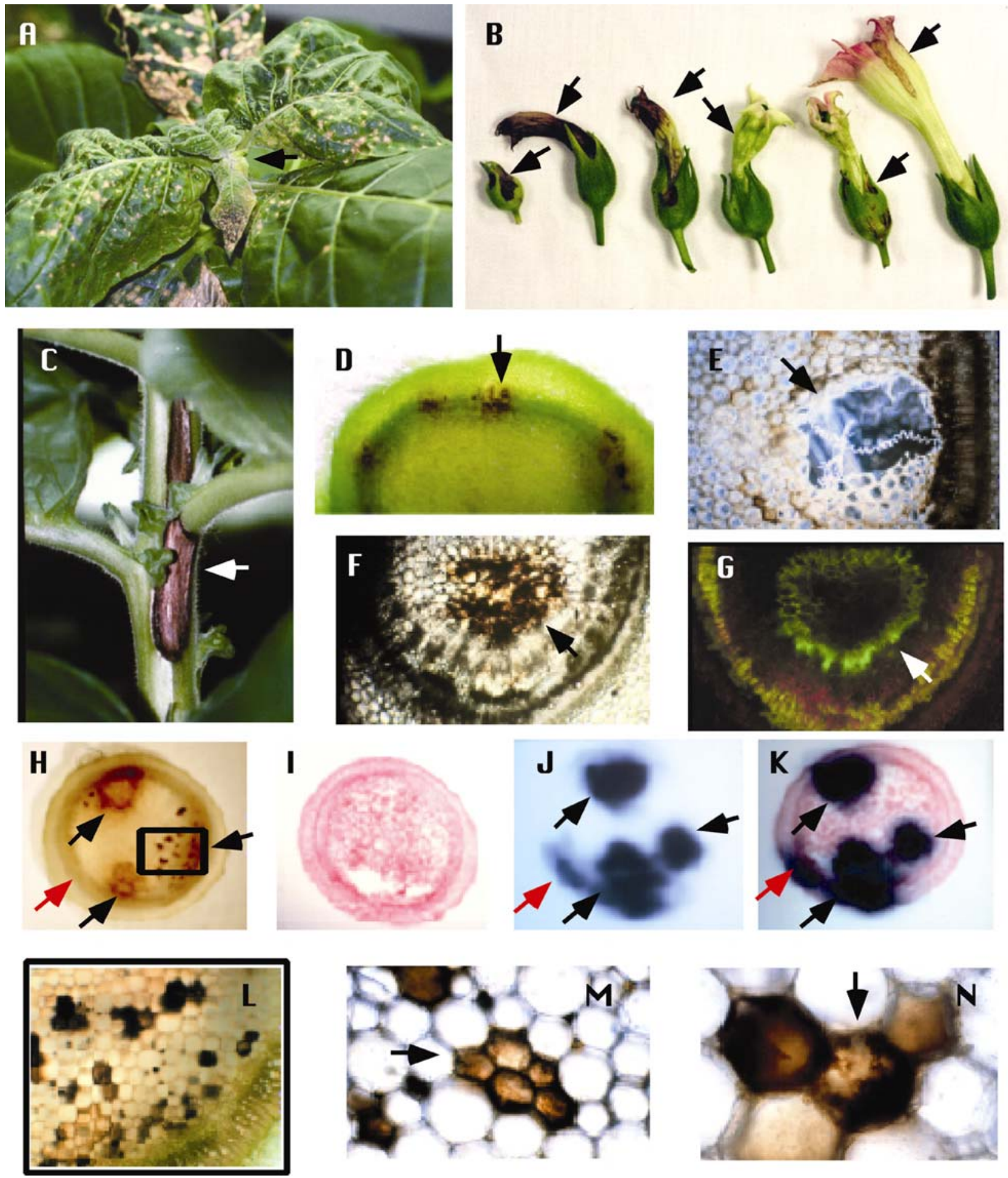

Fig. 4. Plants expressing p35 allow systemic spreading of Tobacco mosaic virus (TMV) in a resistant tobacco plant (NN) background. A, Plant showing systemic hypersensitive response (SHR) lesions and symptoms of systemic TMV infection in upper noninoculated tissue 7 to 10 days after TMV infection in lower leaves. B, Flowers at different stages of development show SHR lesions in different floral tissues as well as symptoms of systemic TMV infection. C, HR-like lesion in the stem about two weeks after inoculation of the lower leaves with TMV. D, Stem transversal section showing browning tissue containing collapsed cells in and around the vascular tissue ( $2 \times$ magnification). E, Hole in the pith, showing the terminal stage of an internal HRlike lesion ( $2 \times$ magnification). F, Transversal section of a stem showing collapsed cells in the pith. G, Same section as in F showing autofluorescence associated with the periphery of the lesion. $\mathbf{H}$, Transversal section of a stem showing different HR-like lesions (1.5× magnification). Black arrows show HR-like lesions. Red arrow shows a symptomless area of the stem that contains TMV (J and K). I, Ponceau S staining of the tissue print obtained with the stem section in H. J, Autoradiograph from a Western blot performed with the printing shown in I using TMV CP antibody. K, I and J superimposed, showing that HR-like lesions correlate with TMV presence, but in some cases, TMV presence does not correlate with obvious symptoms (red arrow). L, $4 \times$ magnification of the boxed area in $\mathrm{H}$ showing isolated or small groups of dying cells. M, Higher magnification (10×) of a group of dying cells. $\mathbf{N}$, Dying cells show accumulation and precipitation of dark material (20× magnification). 
indistinguishable from WT plants and did not show TMV spreading. The lack of HR cell death inhibition in p35mexpressing tobacco plants is likely due to the fact that this mutant form of $\mathrm{p} 35$ protein is unable to form a stable inhibitory complex with one or more putative plant proteases. We obtained an intermediate phenotype with p35D87A-expressing transgenic plants upon infection with TMV to induce the HR. This could be due to residual caspase-inhibiting activity of the p35D87A protein. Indeed, when very high p35D87A expressors were infected with TMV, the phenotype resembled p35-expressing plants in the delay of HR cell death. The integrity of the p35 structure is critical for its function as a caspase inhibitor, as it has been shown by correlation between mutational disruption of defined secondary structures and loss of p35 function (Fisher et al. 1999; Xu et al. 2001). An insertion located within the $C$-terminal $\beta$-sheet (in290) caused loss of function. In line with this observation, we also found that the p35SpeI mutant, which is affected in the $\mathrm{C}$-terminal $\beta$-sheet, does not show any detectable inhibitory effect on HR cell death. This suggests that the overall structure of p35 is crucial for its function as a HR inhibitor in transgenic plants, as it is critical for caspase inhibition in insect cells (Hershberger et al. 1992). In conclusion, lack of HR cell death inhibition in transgenic plants expressing three different mutant forms of the p35 protein (two within the active site and the other one in the C-terminus) confirmed that the HR cell death inhibition shown in p35-expressing plants is likely related to its ability to inhibit caspases in animal systems.

\section{Inhibition of HR cell death leads to systemic spread of viral pathogen in spite of resistance induction.}

The actual contribution of cell death within the HR for pathogen resistance is still a matter of debate. This results from the complexity found in the resistance response (which consists of coordinated actions by multiple factors and pathways) and the diversity in the types of host-pathogen interaction. In addition, the role of cell death within the HR has proved difficult to ascertain, since it is not trivial to uncouple the two phenomena. An example in which disease resistance to bacterial pathogen is displayed in the absence of macroscopic cell death is the dndl mutant of Arabidopsis thaliana (Yu et al. 1998). However, this mutant displays significant levels of salicylic acid and PR expression in the absence of pathogen challenge, which may suggest that constitutive SAR is induced in this mutant and it will be difficult to assess the relative importance of cell death in disease resistance.

Plants expressing p35 represent a novel system in which cell death induction is suppressed, while the rest of the components for resistance response is apparently not affected. Indeed, p35 transgenic plants express PRs at the same rate and level as in WT plants upon infection with TMV, yet these plants show systemic spreading of the virus. A striking aspect shown in the p35-expressing plants is that in spite of the fact that the SAR is likely switched on after TMV infection, the virus is able to induce SHR. Plant viruses enter plant cells, replicate their genome, and spread to adjacent cells through the plasmodesmata (Gilbertson and Lucas 1996). During incompatible interactions, the host is able to prevent the systemic spreading of the virus, localizing the pathogen at the primary site of infection. It has been shown that in plant cells undergoing cell death, the protoplast condenses, resulting in retraction of the plasma membrane from the cell wall and the subsequent severance of plasmodesmatal links, due to water loss (Yano et al. 1998). Partial cell death inhibition in $\mathrm{p} 35$-expressing plants appeared to be enough and sufficient to allow the virus to escape from the primary infection site and infect the plant systemically within a resistant background. A possible interpretation of our results could be that incomplete or delayed cell death in p35-expressing plants maintains, at least to some extent, the protoplast structure and the plasmodesmatal links, keeping physical connection between cells long enough to allow virus spreading during the HR. Thus, it appears that, in this particular plantpathogen interaction (N/TMV), timely and full induction of cell death is necessary to insure restriction of TMV mobility by physically isolating the infected cells.

Our observations with p35-expressing transgenic tobacco plants parallel those reported with mutants of the $N$ gene, some of which interact in a dominant negative manner with the wildtype $\mathrm{N}$ protein (Dinesh-Kumar et al. 2000). In these cases, such as deletion of the Toll-interleukin-1 receptor-like domain or point mutations in the nucleotide-binding site of $\mathrm{N}, \mathrm{TMV}$ infection results in SHR that can spread throughout the infected tobacco plant. Similarly, an earlier study of determinants on the cucumber mosaic virus (CMV) sequence for HR and resistance induction in cowpea revealed that a point mutation at position 1,978 of the CMV 2a polymerase gene can result in systemic $\mathrm{HR}$ as well as systemic infection by CMV (Kim and Palukaitis 1997). However, in neither of these studies were the effects of the mutations on cell death activation rate measured during the HR after the initial infection. Our present study suggests that the phenotypes observed in these instances of either mutations in the $R$ gene (i.e., the tobacco $N$ gene) or the $A v r$ determinant on the pathogen (i.e., the 2 a polymerase gene of CMV) may result from slower rates of PCD induction.

\section{Evolutionary conservation of cell death mechanisms: Divergence or convergence?}

Synthetic and physiological caspase inhibitors affect the progression of HR cell death and other apoptotic processes in plants (De Jong et al. 2000; del Pozo and Lam 1998; Elbaz et al. 2002; Hansen 2000; Lam and del Pozo 2000). Lincoln and associates (2002) recently reported that p35 expression in transgenic tomatoes blocked AAL (Alternaria alternata) toxin-induced cell death and provided protection against the pathogen Alternaria alternata f. sp. lycopersici. In addition, caspase-specific cleavage activity has been detected in plant extracts that were developing a HR (del Pozo and Lam 1998) and in heat-treated tobacco cells (Tian et al. 2000). In vivo caspase-like activity has been characterized in barley and Chara and active caspase-like proteins were detected in whole plant cells undergoing PCD (Elbaz et al. 2002; Korhout at al. 2000). These results and our present data suggest the participation of caspase-like proteases during cell death in plants. So far, no caspase orthologue has been retrieved from any plant genomic database. What is the nature of this putative plant caspase-like protein? There are two possibilities. One possibility is that caspases are evolutionarily conserved and one or a few structural orthologues of animal caspases exist in the plant genome. The fact that these have not been identified yet may indicate that they are not highly homologous in sequence with the animal caspases but may share similar structural protein characteristics. The other possibility is that one or more plant proteases inhibited by peptide inhibitors such as YVAD and the baculovirus p35 protein may be structurally unrelated to animal caspases. In this case, the one or more plant proteases involved in controlling HR cell death must have evolved to cleave relevant target sites of similar specificity as those of animal caspases. In the case of the $\mathrm{p} 35$ protein, our present results suggest that similar structure in the active site of these one or more plant proteases must have evolved to resemble that of caspases. Database searches revealed the presence of putative caspase-like proteins in the genome of Dictyostelium discoideum, Streptomyces coelicolor, and Rhizobium sp. (Aravind et al. 1999), and more recent structural element-based iterative searches have uncovered cas- 
pase-related protease families designated as paracaspases and metacaspases (Uren et al. 2000). The latter work revealed that the caspase domain belongs to an ancient protease superfamily with metacaspases represented in yeast and plant genomes. Indeed, the single metacaspase gene YCAl in yeast has recently been shown to possess caspase-like protease activities with distinct substrate specificity (Madeo et al. 2002). Furthermore, ycal mutants display a dramatic suppression of cell death induced by oxidative stress, thus providing direct genetic evidence that the yeast metacaspase YCA1 can be a regulator of cell death activation in an analogous manner to the animal caspases. Whether structurally conserved regulators such as the plant metacaspases are used to orchestrate the HR during plant defense response will shed light on the evolutionary origin of PCD in plants and animals. In any case, our present results show that, like animal cells, plants also deploy cell death as a containment mechanism in order to restrict the proliferation of viral invaders within the organism.

\section{MATERIALS AND METHODS}

Plant material and infection with pathogens.

TMV strain U1 infection and $P$. syringae pv. phaseolicola NPS 3121 or NPS 4000 were prepared and infiltrated in tobacco plants as described by del Pozo and Lam (1998). Bacteria was used at $0.5 \times 10^{8} \mathrm{CFU}$ per ml. For measurement of ion leakage, five leaf discs (9-mm diameter) per plant were used for TMV experiments, and three leaf discs (11-mm diameter) were used for bacteria experiments. In both cases, leaf discs were floated on $3 \mathrm{ml}$ of milliQ water at room temperature for 6 $\mathrm{h}$ under gentle agitation. Column values represent the average of three plants per line. The conductivity of the bathing solution was measured with a conductivity meter (model 604, VWR Scientific, Mississauga, Ontario, Canada). Each experiment was performed at least three times.

\section{RNA and protein isolation and analysis.}

The procedures for RNA isolation, Northern blot, and Western blot analysis of proteins were essentially as described previously (Mittler et al. 1995).

\section{Construction of transgenic tobacco plants.}

p35 coding sequence was subcloned from pBS-P35 (Clem et al. 1996) as a BamHI/SstI fragment and was inserted into the binary vector EL103 (Fig. 1A) (Mittler et al. 1995). The p35SpeI mutant was generated by cutting pBS-P35 (Clem et al. 1996; Hershberger et al. 1992) with BamHI and SpeI that generates a fragment that is lacking 12 amino acid residues at the $\mathrm{C}$-terminus. The insert was subcloned into the binary expression vector EL103 cut with BamHI/XbaI by using an adaptor (SpeI/XbaI) that introduces a stop codon at the $3^{\prime}$-terminus of the p35 coding sequence. For the construction of p35m, pBS$\mathrm{P} 35$ was used as a template in two polymerase chain reaction (PCR) reactions with the following primer pairs: $\mathrm{T} 3$ and EL666 (5'TACAGCGCTCAAATGGCTGG3') and T7 and EL665 (5'CATTTGAGCGCTGTAAGCGCG3'). The mutations introduce a new HaeII restriction site in nucleotide position 457 of the coding region. PCR products were subcloned into pBluescript-SK (pSK) cut with EcoRV to generate EL666/T3-pSK and EL665/T7-pSK plasmids. Both were sequenced to confirm the lack of PCR-generated mutations. Ligating the purified fragments, EL666/T3 (BamHI/HaeII) and EL665/T7 (HaeII/SstI), into the binary vector EL103 (BamHI/XbaI) created the final construct. p35D87A coding sequence was subcloned from pDX1 (Xue and Horvitz 1995) as a XhoI/BamHI fragment in the binary vector EL103 (BamHI/SstI) by using an XhoI/SstI adaptor.
In vitro translation

of p35 and p35 mutants and caspase cleavage assay.

p35, p35m, and p35D87A fragments were cut with EcoRI at the $5^{\prime}$ end and with SpeI at the $3^{\prime}$ end, resulting in a fragment that lacks 12 amino acid residues at the C-terminus. These fragments were ligated into the bacterial expression vector $\mathrm{pET}$ 22b (Novagen, Madison, WI, U.S.A.) (EcoRI/XhoI) by using an adaptor SpeI/XhoI, which restores the C-terminus coding region of $\mathrm{p} 35$, in order to generate in-frame p35 His-tagged clones. For pET22b/p35SpeI, the same strategy was followed, using a shorter SpeI/XhoI adaptor that did not restore the p35 coding region at the $\mathrm{C}$-terminus. In vitro translated $\left[{ }^{35} \mathrm{~S}\right]$-methionine-labeled p35, p35m, p35D87A, and p35SpeI proteins were obtained using the TNT coupled reticulocyte lysate system (Promega, Madison, WI, U.S.A.) following the manufac-

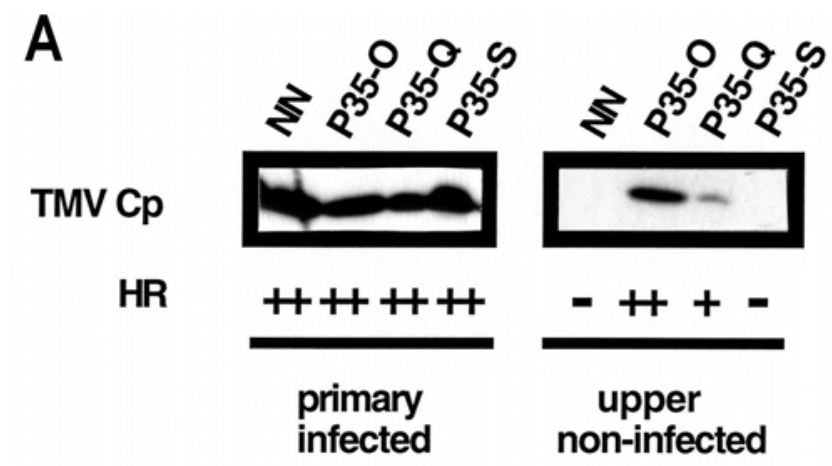

B

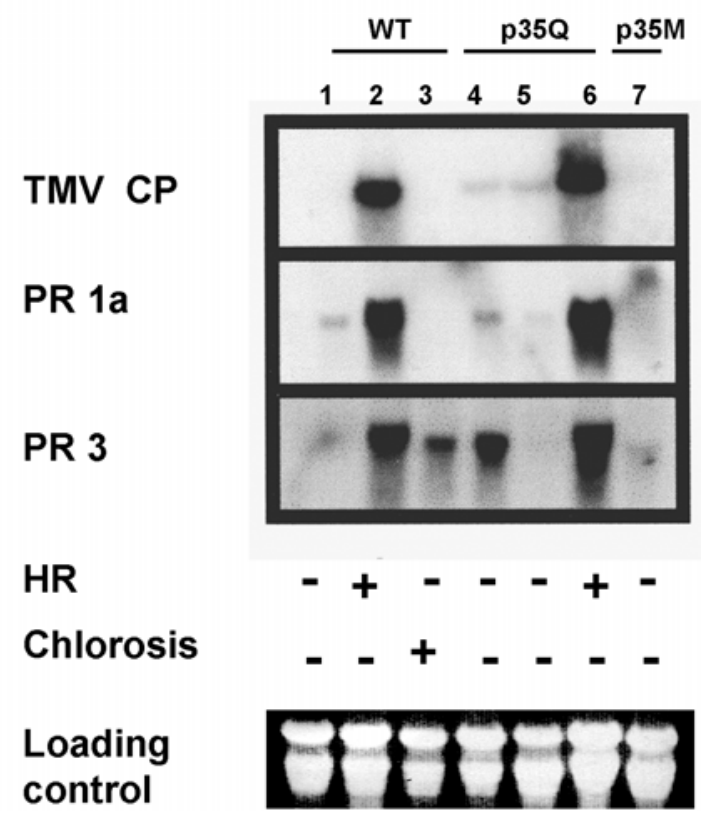

Fig. 5. A, Lesion formation correlates with Tobacco mosaic virus (TMV) presence. Tissues were collected from primary TMV-infected leaves of wild-type (WT) Samsun $N N$ tobacco plants and three independent lines of transgenic plants expressing p35 (p35-O, p35-Q, and p35-S). Tissues from upper uninoculated leaves between two and three weeks after infection of the lower leaves were also analyzed. (-) no visible hypersensitive response (HR), (+) few HR lesions, and (++) severe HR. B, TMV presence in systemic tissues correlates with pathogenesis-related gene expression in p35-expressing plants. Northern blot performed with tissue collected from WT tobacco leaves before infection (1), primary infected leaves (2), upper uninfected leaves (3), and systemic leaf samples from $\mathrm{p} 35-\mathrm{Q}$ and $\mathrm{p} 35-\mathrm{M}$ lines showing different symptoms of TMV infection (lanes 4, 5, 6, and 7). The blot was hybridized with the probes indicated on the left. A picture of the membrane after transfer is shown on the bottom to illustrate the similar amount of RNA loaded for each sample. 
turer's suggestions. Caspase cleaving assay was performed by adding $10 \mathrm{ng}$ of purified caspase- 1 in caspase reaction buffer (del Pozo and Lam 1998) and by incubating the solution for 30 min at $30^{\circ} \mathrm{C}$. The percentage of cleavage was determined by phosphorimager analysis using the ImageQuant program (Molecular Dynamics, Sunnyvale, CA, U.S.A.) after separation of the proteins by sodium dodecyl sulfate-polyacrylamide gel electrophoresis.

\section{Tissue print.}

Tissue printing was performed using $0.2 \mu \mathrm{m}$ nitrocellulose membrane (Bio-Rad, Hercules, CA, U.S.A.) following the directions of Almoguera and associates (1993). Western blot of the prints was performed as described before (Mittler et al. 1995), using TMV CP antisera.

\section{ACKNOWLEDGMENTS}

We thank L. Miller (Athens, GA, U.S.A.) for the p35 gene and anti-p35 antisera; R. Horvitz and D. Xue (Boston) for the p35D87A gene; D. Klessig (Ithaca, New York, U.S.A.) for the $P R$ gene probes, R. Talanian for the purified caspase-1 protein, and N. Tumer for the TMV CP antisera. Critical comments on the manuscript by D. Pontier are also gratefully acknowledged. This work was supported in part by the New Jersey Commission of Science and Technology. O. del Pozo was supported in part by a postdoctoral fellowship (F.P.I.) from the Spanish Ministry of Science and Education.

\section{LITERATURE CITED}

Almoguera, C., Coca, M. A., and Jordano, J. 1993. Tissue-specific expression of sunflower heat shock proteins in response to water stress. Plant J. 4:947-958.

Aravind, L., Dixit, V. M., and Koonin, E. 1999. The domains of death: Evolution of the apoptosis machinery. TIBS 24:47-53.

Bertin, J., Mendrysa, S. M., LaCount, D. J., Gaur, S., Krebs, J. F., Armstrong, R. C, Tomaselli, K. J., and Friesen, P. D. 1996. Apoptotic suppression by baculovirus P35 involves cleavage and inhibition of a virus-induced CED-3/ICE-like protease. J. Virol. 70:6251-6259.

Bump, N. J., Hackett, M., Hugunin, M., Seshagiri S., Brady, K., Chen, P., Ferenz, C., Franklin S., Ghayur, T., Li, P., Licari, P., Mankovich, J., Shi, L., Greenberg, A. H., Miller, L. K. and Wong, W. W. 1995. Inhibition of ICE family proteases by baculovirus antiapoptotic protein p35. Science 269:1885-1888.

Clem, R. J., Hardwick, M. J., and Miller, L. K. 1996. Anti-apoptotic genes of baculoviruses. Cell Death Differ. 3:9-16.

Cole, A. B., Kiraly, L., Ross, K., and Schoelz, J. E. 2001. Uncoupling resistance from cell death in the hypersensitive response of Nicotiana species to Cauliflower mosaic virus infection. Mol. Plant-Microbe Interact. 14:31-41.

Cryns, V., and Yuan, J. 1998. Proteases to die for. Genes Dev. 12:15511570.

De Jong, A. J., Hoeberichts, F. A., Yakimova, E. T., Maximova, E., and Woltering, E. J. 2000. Chemical-induced apoptotic cell death in tomato cells: Involvement of caspase-like proteases. Planta 211:656-62.

del Pozo, O., and Lam, E. 1998. Caspases and programmed cell death in the hypersensitive response of plants to pathogens. Curr. Biol. 8:11291132 .

Dinesh-Kumar, S. P., Tham, W.-H., and Baker, B. J. 2000. Structure-function analysis of the tobacco mosaic virus resistance gene $N$. Proc. Natl. Acad. Sci. U.S.A. 97:14789-14794.

Elbaz, M., Avni, A., and Weill, M. 2002. Constitutive caspase-like machinery executes programmed cell death in plant cells. Cell Death Differ. 9:726-733.

Fisher, A. J., de la Cruz, W., Zoog, S. J., Schneider, C. L., and Friesen, P. D. 1999. Crystal structure of baculovirus P35: Role of a novel reactive site loop in apoptotic caspase inhibition. EMBO (Eur. Mol. Biol. Organ.) J. 18:2031-2039.

Gilbertson, R. L., and Lucas, W. J. 1996. How do viruses traffic on the 'vascular highway'? Trends Plant Sci. 1:260-267.

Greenberg, J. T. 1996. Programmed cell death: A way of life for plants.
Proc. Natl. Acad. Sci. U.S.A. 93:12094-12097.

Hansen, G. 2000. Evidence for Agrobacterium-induced apoptosis in maize cells. Mol. Plant-Microbe Interact. 13:649-657.

Hershberger, P. A., Dickson, J. A., Friesen, P. D. 1992. Site-specific mutagenesis of the 35-kilodalton protein gene encoded by Autographa californica nuclear polyhedrosis virus: Cell line specific effects on virus replication. J. Virol. 66:5525-5533.

Kidd, V. J. 1998. Proteolytic activities that mediate apoptosis. Annu. Rev. Physiol. 60:533-573.

Kim, C.-H., and Palukaitis, P. 1997. The plant defense response to cucumber mosaic virus in cowpea is elicited by the viral polymerase gene and affects virus accumulation in single cells. The EMBO (Eur. Mol. Biol. Organ.) J. 16:4060-4068.

Korhout, A. A. J., Berecki, G., Briun, W., van Duijn, B., and Wang, M. 2000. The presence and subcellular localization of caspase 3-like proteinases in plant cells. FEBS (Fed. Eur. Biochem. Soc.) Lett. 475:139-144.

Lam, E., and del Pozo, O. 2000. Caspase-like protease involvement in the control of plant cell death. Plant Mol. Biol. 44:417-428.

Lincoln, J. E., Richael, C., Overduin, B., Smith, K., Bostock, R., and Gilchrist, D. G. 2002. Expression of the antiapoptotic p35 gene in tomato blocks program cell death and provides broad-spectrum resistance to disease. Proc. Natl. Acad. Sci. U.S.A. 99:15217-15221.

Lindgren, P. B., Peet, R. C., and Panopoulos, N. J. 1986. Gene cluster of Pseudomonas syringae pv. "phaseolicola" controls pathogenicity of bean plants and hypersensitivity on nonhost plants. J. Bacteriol. 168:512-522.

Madeo, F., Herker, E., Maldener, C., Wissing, S., Lachelt, S., Herlan, M., Fehr, M., Lauber, K., Sigrist, S. J., Wesselborg, S., and Frohlich, K.-U. 2002. A caspase-related protease regulates apoptosis in yeast. Mol. Cell 9:911-917.

Mittler, R., and Lam, E. 1996. Sacrifice in the face of foes: Pathogen-induced programmed cell death in plants. Trends Microbiol. 4:10-15.

Mittler, R., Shulaev, V., and Lam, E. 1995. Coordinated activation of programmed cell death and defense mechanisms in transgenic plants expressing a bacterial proton pump. Plant Cell 7:29-42.

Morel, J. B., and Dang1, J. L. 1997. The hypersensitive response and the induction of cell death in plants. Cell Death Differ. 4:671-683.

Raff, M. 1998. Cell suicide for beginners. Nature 396:119-122.

Schotte, P., Declercq, W., VanHuffel, S., Vandenabeele, P., and Beyaert, R. 1999. Non-specific effects of methy ketone inhibitors of caspases. FEBS (Fed. Eur. Biochem. Soc.) Lett. 442:117-121.

Tian, R.-H., Zhang, G.-Y., Yan, C.-H., and Dai, Y.-R. 2000. Involvement of poly(ADP-ribose) polymerase and activation of caspase-3-like protease in heat shock-induced apoptosis in tobacco suspension cells. FEBS (Fed. Eur. Biochem. Soc.) Lett. 474:11-15.

Uren, A. G., O’Rourke, K., Aravind, L., Pisabarro, T. M., Seshagiri, S., Koonin, E. V., and Dixit, V. M. 2000. Identification of paracaspases and metacaspases: Two ancient families of caspase-like proteins, one of which plays a key role in MALT lymphoma. Mol. Cell 6:961-967.

Van der Biezen, E. A., and Jones, J. G. N. 1998. The NB-ARC domain: A novel signalling motif shared by plant resistance gene products and regulators of cell death in animals. Curr. Biol. 7:226-227.

Whitman, S., Dinesh-Kumar, S. P., Choi, D., Hehl, R., Corr, C., and Baker, B. 1994. The product of the tobacco mosaic virus resistance gene $N$ : Similarity to toll and the interleukin-1 receptor. Cell 78:11011115 .

Xu, G., Cirill, M., Huang, Y., Rich, R. L., Myzska, D. G., and Wu, H. 2001. Covalent inhibition revealed by the crystal structure of the caspase-8/p35 complex. Nature 410:494-496.

Xue, D., and Horvitz, H. R. 1995. Inhibition of the Caenorhabditis elegans cell death protease CED-3 by a CED-3 cleavage site in baculovirus p35 protein. Nature 377:248-251.

Yang, Y., Shah, J., and Klessig, D. 1997. Signal perception and transduction in plant defense responses. Genes Dev. 11:1621-1639.

Yano, A., Suzuki, K., Uchimiya, H., and Shinshi, H. 1998. Induction of hypersensitive cell death by a fungal protein in cultures of tobacco cells. Mol. Plant-Microbe Interact. 11:115-123.

Yu, I. C., Parker, J., and Bent, A. F. 1998. Gene-for gene disease resistance without the hypersensitive response in the Arabidopsis dnd1 mutant. Proc. Natl. Acad. Sci. U.S.A. 95:7819-8724.

Zhou, Q., Krebs, J. F., Snipas, S. J., Price, A., Alnemri, E. S., Tomaselli, K. J., and Salvesen, G. S. 1998. Interaction of the baculovirus antiapoptotic protein p35 with caspases. Specificity, kinetics and characterization of the caspase/p35 complex. Biochemistry 37:10757-10765. 